\title{
Two families with the Li-Fraumeni cancer family syndrome
}

\author{
A D J PEARSON,* A W CRAFT, ${ }^{*}$ J M RATCLIFFE, $\dagger$ J M BIRCH, $\uparrow$ P MORRIS-JONES, $\dagger$ \\ AND D F ROBERTS* \\ From * the Departments of Child Health and Human Genetics, University of Newcastle upon Tyne \\ and Royal Victoria Infirmary, Newcastle upon Tyne; and the Departments of Child Health and \\ Epidemiology and Social Research, University of Manchester, Manchester.
}

SUmmary The first two families to be identified in the United Kingdom with the Li-Fraumeni syndrome of familial cancer are reported. The first family comprises breast carcinoma in the mother and adrenocortical carcinoma, medulloblastoma, and rhabdomyosarcoma in three of her four children, and the second family comprises breast carcinoma in the mother and adrenocortical carcinoma and rhabdomyosarcoma in two of her three children. All three of the surviving children with malignancy, and one other who has recently died, possess the tissue type antigen B12.

Childhood cancer is not evenly distributed among the population and familial aggregations sometimes occur. Much of this familial clustering can be explained by known heritable diseases, for example, some retinoblastomas and the frequency of malignancy in neurofibromatosis. In addition there seem to be occasional families with a predisposition to various types of malignancy, the so-called 'cancer families', and nine such families in the United States have been reported. ${ }^{1-7}$ We report two similar families from the United Kingdom.

The families were identified through the population based Children's Malignant Disease Registries of the Northern and North-Western Regions. ${ }^{89}$ In the Northern Region between 1968 and 1980 there have been five other sib pairs out of a total of 1100 cases notified to the Registry. Three of these each had bilateral retinoblastoma but the other two were apparently unrelated malignancies with acute lymphoblastic leukaemia (ALL) and retinoblastoma, and ALL and medulloblastoma. In the Manchester Children's Tumour Registry there have been seven other sib pairs among a total of 2702 cases of malignant tumours registered from 1954 to 1980 . These included two sib pairs of bilateral retinoblastoma.

\section{Case reports}

The immediate family pedigrees are shown in figs 1 and 2.

FAMILY A

The mother was born on 27.7.41. At the age of 33, 2 years after the birth of her fourth child, she Received for publication 17 March 1982 presented with a mass in the right breast. An infiltra- $\vec{\varnothing}$ ting and intraduct poorly differentiated adenocarci- ? noma of the breast was found and, despite treatment, she died 4 years later with metastatic disease in liver and bone. There was no necropsy. She had been a non-smoker.

The father was born on 22.7.36. He is a healthy, 45year-old, non-smoking office worker.

The first child was born on 29.9.63. She presented at the age of 22 months with signs of virilisation. Excessive levels of urinary hydroxy- and ketosteroids suggested the presence of an adrenal tumour, and this was confirmed at laparotomy where two well encapsulated adrenal tumours were removed from the left side, the right adrenal appearing normal. Histology showed this to be an adrenocortical carcinoma. She has had no further treatment and, at

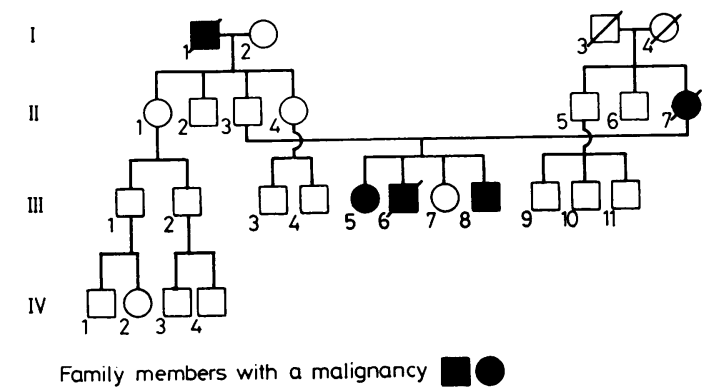

FIG 1 Pedigree of family A. I.1 gastric carcinoma, II.7 breast carcinoma, III.5 adrenocortical carcinoma, III.6 medulloblastoma, III.8 rhabdomyosarcoma. 


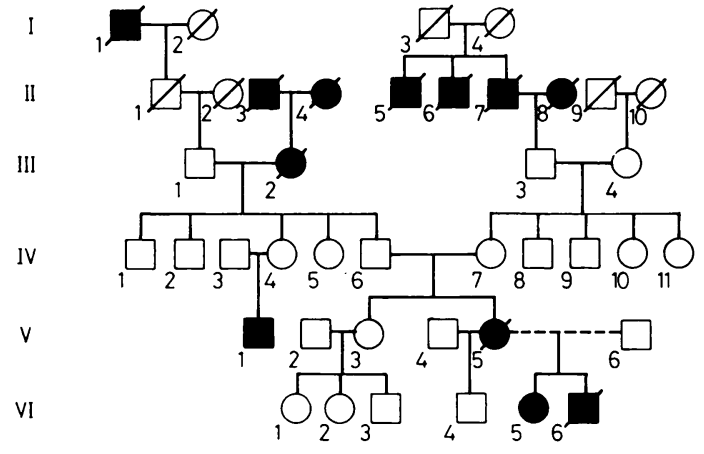

Family members with a malignancy

FIG 2 Pedigree of family B. I.1 carcinoma of lung, II. 3 carcinoma of lung, II.4 carcinoma unknown site, II.5 carcinoma of colon, II.6 carcinoma of stomach, II.7 carcinoma of lung, II.8 carcinoma of stomach, III. 2 carcinoma of breast, V.I testicular seminoma, V.6 carcinoma of breast, VI.5 adrenocortical carcinoma, VI.6 rhabdomyosarcoma.

the age of 18 , is a normal post-pubertal girl with no evidence of recurrence of her tumour.

The second child was born on 18.9.67. At the age of $3 \frac{1}{2}$ he presented with signs of ataxia and raised intracranial pressure. At craniotomy a right cerebellar medulloblastoma was subtotally removed. Despite postoperative radiotherapy he died at the age of 4 , 6 months after diagnosis. There was no necropsy.

The third child was born on 11.6.71 and is a normal prepubertal 10-year-old girl.

The fourth child was born on 12.6.72. He presented at the age of 3 years with a hard swelling in his right forearm and biopsy showed this to be a rhabdomyosarcoma. An above elbow amputation was performed and vincristine, actinomycin $\mathrm{D}$, and cyclophosphamide were given for 2 years. He remains well 5 years after presentation with no evidence of recurrent disease.

A further 182 family members have been traced and among these there were two of 43 paternal relatives and two of 139 maternal relatives with cancer. These were the paternal grandfather with gastric carcinoma at 66 years, a paternal uncle once removed (a smoker) with an oat cell carcinoma of the bronchus at 35 years, a maternal fourth degree relative with a colonic carcinoma at the age of 64 years, and a maternal great aunt with a renal carcinoma at the age of 38 years.

FAMILY B

The mother was born on 8.2.53 and has been married three times, with one child by her first husband and two by her second. At the age of 27,3 months after the birth of her third child, she presented with a mass in her left breast. A poorly differentiated adenocarcinoma was confirmed at biopsy. Despite mastectomy and local radiotherapy she died a year later with widespread metastases. There was no necropsy.

The father was born on 17.12.51. He is a healthy, 29-year-old manual worker.

The first child by the first marriage was born on 18.12.71 and is a healthy 10-year-old boy.

The second child was born on 20.7.78. At the age of 16 months she presented with signs of virilisation. Investigations suggested an adrenal tumour and at laparotomy a well encapsulated left adrenal tumour was removed. Histology showed this to be an adrenal cortical tumour. Sixteen months later she has no evidence of recurrent disease.

The third child was born on 20.10.79. He presented at the age of 20 months with a progressive swelling of the right periauricular area, following which the tumour appeared extending from the middle ear. Biopsy showed this to be a botryoid rhabdomyosarcoma. He responded well to the first 4 months of chemotherapy, but then relapsed with widespread metastases and died.

Another unusual tumour has occurred in this family in the mother's cousin. This boy presented at the age of 22 years with a testicular seminoma and remains well with treatment. There are another eight family members with malignancy, but all occurred over the age of 60 . Extensive tracing of family members, however, has not yet been completed.

\section{Further family studies}

The HLA types of the surviving family members and

TABLE HLA typing.

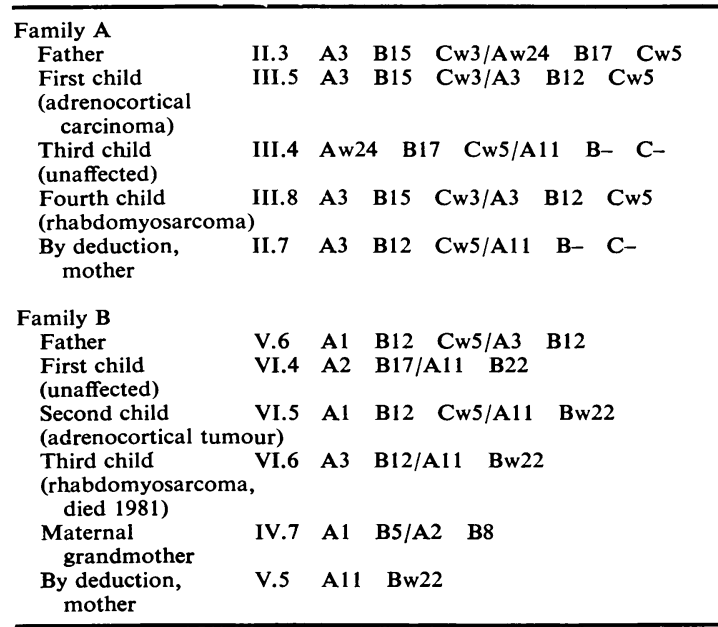


one child who has now died are shown in the table. The first and fourth children in family A (as well as being identical at HLA loci A, B, and C) are mutually non-responsive in mixed lymphocyte culture suggesting that they are also $D$ locus identical.

Chromosome analysis of the peripheral blood leucocytes of the same surviving family A members showed no evidence of chromosomal damage, aneuploidy, fragile sites, or banding polymorphism. Markers (specific satellites) for a chromosome 14 were present in the father and child 4 , and for a chromosome 21 in the father and child 3 and 4.

\section{Discussion}

It has previously been reported by Draper et al ${ }^{10}$ that even when known inherited diseases are excluded, there is an increased risk of other sibs developing malignancy if one child presents with cancer, the risk being 1 in 300 as compared with 1 in 600 for the general population. Some of this increase may be accounted for by a genetic component. We believe families $\mathrm{A}$ and $\mathrm{B}$ are examples of the $\mathrm{Li}$ Fraumeni syndrome. This was first described in 1969 in four families in which sibs or cousins had a rhabdomyosarcoma and there was malignancy at a young age in one of the parents. ${ }^{1}$ This constellation of tumours was extended to include brain tumour and adrenocortical carcinoma ${ }^{2}$ and later leukaemia ${ }^{3}$ in children, and other adult tumours. ${ }^{4}$ The characteristics of the syndrome appear to be an aggregation of childhood tumours with an increased incidence of malignancy in young adult family members. Both the child and the adult malignancies usually present at a younger age than that of the general population. There is also a tendency for multiple primary neoplasms and the second tumour tends to be of the same type as that in the sib. ${ }^{1}$ It has been suggested that an autosomal dominant gene with variable expressivity, modified perhaps by an environmental agent, may be responsible and that the agent is an oncogenic virus. This could explain the explosive nature, in time, of the syndrome ${ }^{5}$ which is very apparent in both the families, with all the malignancies presenting within 9 years in family $A$ and within 19 months in family $B$.

Family A shows a very compact manifestation of the syndrome, although there is no increased incidence of malignancies in the extended family. The four identified members with malignancy are not significantly different from the nine which would be expected as calculated from Serial Mortality Tables. ${ }^{11}$ This suggests that if there is dominant transmission the mother may have possessed a new mutant gene. The absence of chromosomal abnormalities in the surviving members of the family conflict with the findings of Bottomley et $\mathrm{al}^{3}$ of an increased incidence $\stackrel{\overparen{P}}{\stackrel{4}{+}}$ of aneuploidy in the cultured peripheral blood $\overrightarrow{\bar{B}}$ leucocytes of the family.

Although HLA typing has been performed in other cancer family syndromes, ${ }^{12}$ it has not been reported in families with the $\mathrm{Li}$-Fraumeni syndrome. There appeared to be no common HLA haplotype but the antigen B12 occurred in all three surviving $\mathrm{c}$ members with a malignancy and one who has $\overrightarrow{0}$ subsequently died, and by deduction must have occurred in the mother in family A.

Although the antigen B12 occurs in $30 \%$ of the population, it has not previously been described as being associated with any neoplasm. However, there $\vec{\omega}$ is an increased incidence of the antigen B12 in the ir longer term survivors of acute myeloid leukaemia. ${ }^{13} \mathrm{\omega}$ Although certain antigens (A24, A28, A29, B15, N B35) have been seen with increased incidence in $\frac{\text { ? }}{2}$ families prone to breast cancer, ${ }^{12}$ no definite associa- $\vec{O}$ tion has been proved. It would be of interest if B12 $ᄋ$ occurred in members of other families with the Li- 0 Fraumeni syndrome and if children with adreno- 므 cortical carcinoma associated with the syndrome $\vec{\theta}$ possessed the antigen, while sporadic cases did not.

Adrenocortical carcinoma is extremely rare, accounting for only $0.2 \%$ of all childhood malignancies, ${ }^{10}$ but it is over-represented in families with more than one childhood malignancy and as a second $\bar{\partial}$ primary tumour. ${ }^{1314}$ Its occurrence should alert $\frac{0}{Q}$ clinicians to the possibility of this being a manifesta- $\triangle$ tion of a family cancer syndrome.

The importance of 'cancer families' is twofold. Firstly, they may prove to be a cornerstone in the investigation of the aetiology of malignancy, in particular the interplay of inherited and environmental factors. Secondly, having identified the families, they pose a challenge in terms of cancer $\frac{5}{3}$ surveillance. Not only may other sibs have an increased chance of malignancy but affected family members may have a risk of developing further primary tumours. Close surveillance and screening $ᄋ$ procedures, especially of the breast, can thus be $>$ directed at a high risk population.

A D J Pearson and J M Ratcliffe are supported by N the Leukaemia Research Fund. We are grateful to $\underset{\omega}{N}$ the Tyneside Leukaemia Research Association and 0 the North of England Cancer Campaign for their support of the Northern Region Children's Malig- $\frac{0}{\varnothing}$ nant Disease Registry, and to the Cancer Research $\stackrel{\oplus}{\rightarrow}$ Campaign for support of the Manchester Children's 0 Tumour Registry. We thank the various consultants $\bar{O}$ involved in the care of the families for allowing us to report the families, and the technicians of the $\mathbb{D}$ Department of Human Genetics. 


\section{References}

1 Li FP, Fraumeni JF. Soft tissue sarcomas, breast cancer and other neoplasms. A familial syndrome? Ann Intern Med 1969;71:747-52.

${ }^{2}$ Li FP, Fraumeni JF. Rhabdomyosarcoma in children: epidemiologic study and identification of a familial cancer syndrome. J Natl Cancer Inst 1969;43:1364-73.

3 Bottomley RH, Trainer AI, Condit DT. Chromosome studies in a "cancer family". Cancer $1971 ; 28: 519-28$.

${ }^{4}$ Lynch HT, Mulcahy GM, Harris RE, Guirgis HA, Lynch JF. Genetic and pathological findings in a kindred with hereditary sarcoma, breast cancer, brain tumours, leukaemia, lung, laryngeal and adrenocortical carcinoma. Cancer $1978 ; 41: 2055-64$.

5 Blattner WA, McGuire DB, Mulvihill JJ, Lampkin BC, Hananian J, Fraumeni JF. Genealogy of cancer in a family. JAMA 1979;241:259-61.

6 Lynch HT, Guirgis HA. Childhood cancer and the SBLA syndrome. Med Hypotheses 1979;5:12-22.

7 Lynch HT, Lynch JF, Lynch P. Breast cancer genetics and cancer control. Tumour association. Arch Surg 1975;110: 1227-9.

8 Craft AW. The Northern Regional Children's Malignant Disease Registry. The first ten years. Submitted for publication.
9 Birch JM, Marsden HB, Swindell R. Incidence of malignant disease in childhood; a 24-year review of the Manchester Children's Tumour Registry data. $B r J$ Cancer 1980;42:215-33.

10 Draper GJ, Heaf MM, Kinnier-Wilson LM. Occurrence of childhood cancers among sibs and estimation of familial risks. J Med Genet 1977;14:81-90.

11 Case RAM, Coghill C, Davies JM, et al. Serial mortality tables. In: Neoplastic diseases. Vol 1. England and Wales 1911-1970. London: Division of Epidemiology, Institute of Cancer Research, 1976.

12 Lynch HT, Terasaki PI, Guirgis HA, et al. HLA in breast cancer-prone families and the cancer family syndrome. Prog Clin Biol Res 1977;16:149-62.

13 von Fliedner VE, Sultan Khan Z, Jeannet M. HLA-A and HLA-B antigens in acute leukaemia: A2-B12 phenotypes correlate with longer survival in acute myelogenous leukaemia. Acta Haematol 1981 ;65:73-8.

14 Miller RW. Peculiarities in the occurrence of adrenal cortical carcinoma. Am J Dis Child 1978;123:235-6.

Requests for reprints to Dr A D J Pearson, Department of Child Health, Royal Victoria Infirmary, Newcastle upon Tyne NE1 4LP. 